\title{
Insights of Trends and Developments in Cloud Computing
}

\author{
Dr. Anupam Mazumdar ${ }^{1}$, Husam Alharahsheh2 ${ }^{*}$ \\ ${ }^{1}$ Lecturer at University of Wales Trinity Saint David, College St, Lampeter SA48 7ED, United Kingdom \\ 2Lecturer in business Management at University of Wales Trinity Saint David - London and Senior visiting lecturer across several UK \\ and international Higher Education Institutions, College St, Lampeter SA48 7ED, United Kingdom
}

\section{*Corresponding Author \\ Husam Helmi Alharahsheh}

\section{Article History}

Received: 15.10 .2019

Accepted: 24.10.2019

Published: 04.11.2019

\begin{abstract}
Under the competitive and technology dependent global environment, modern technologies like Cloud Computing are keep boosting. Undoubtedly, enhancements of Cloud Computing are advancing their levels of productivity, profits and gaining market shares. As Cloud Computing already become popular throughout the glove and various peoples from different sectors are using Cloud Computing for different reasons therefore, it is now required to discuss insights of technology trends and developments for Cloud Computing in general. This paper mainly focuses on fundamental understanding of the different aspects of Cloud Computing such as an insight of Cloud Computing, different definitions, characteristics, deployment models, service models, key drivers and key challenges of Cloud Computing.
\end{abstract}

Keywords: Cloud Computing, modern technology, deployment models \& service models.

\section{INTRODUCTION}

Research into information technology adoption and innovation is a constantly expanding area; meanwhile new technologies are evolving on a daily basis [24]. Information technology maintains its insistent march into nearly every aspect of the businesses world [3]. Innovation in information technology makes organization tasks initially hard because of the issues of installation, adoption and implementation of new technologies. This chapter will review Cloud Computing (CC) in general by discussing various aspects such as barriers and incentives to adoption in order to provide a general idea of Cloud Computing opportunities in business. This chapter will further discuss how Cloud Computing can be used in SMEs and what would be the benefits of using it. Moreover, this chapter will further discuss the concept of Digital Bangladesh and how this initiative is associated with Cloud Computing. Finally, this chapter will present the appropriate theories on which the conceptual framework of this research was based -- including a detail review of prior Cloud Computing adoption studies alongside studies of the adoption and use of new technologies.

\section{Cloud Computing - An Insight}

According to [6], "Cloud Computing cannot be a new and innovative technology since CC systems use relatively old computing and network technologies (hardware and software)". Cloud Computing is not an entirely new concept but develops from a long history - perhaps beginning in 'time-sharing' mainframe computer systems and (with the rapid development of local-area computer networks) developing through the 1970's into the technology that came to be known as 'distributed computing'. The first textbooks and college courses on 'distributed computing' appear in the mid-1970's (See Symposium on Principles of Distributed Computing (PODC), 1982) and, with the arrival of network technologies (like Ethernet, from the late 1970's) and more reliable data communications, solving computationally hard problems by sharing programs, data and communications across multiple platforms involving many 'computing nodes' located across a wide-area network became prevalent. ARPANET - the precursor to the Internet was invented in the 1960's and one of its earliest applications was E-Mail - itself an example of large-scale, multi-platform distributed computing across wide area networks. One needs, therefore, to consider more closely what more recent 'Cloud Computing services' offer that sets them apart from their historical predecessors; what makes CC distinctively different from distributed systems? In the following, I suggest that one part of that difference lies in the business model which CC presents for access to and use of distributed (remote) computing services.

Copyright @ 2019: This is an open-access article distributed under the terms of the Creative Commons Attribution license which permits unrestricted use, distribution, and reproduction in any medium for non commercial use (NonCommercial, or CC-BY-NC) provided the original author and source are credited. 
Cloud Computing (CC) represents an on-demand distribution trade model of computing services, which includes "pay-peruse" services. By using this service, service users (clients) pay only for their exploited services, which is similar to the older communal utility amenities/timesharing [6]. Cloud Computing also guarantees to distribute perceptible business reimbursements at lower cost because CC decreases the upfront expenses of computing that most of the time discourages small and medium sized businesses from exploiting fully Information and Communication Technologies (ICT) in their businesses [23]. The services of Cloud Computing offer a reasonable return on the investment of small and medium sized businesses, it is claimed, owing to significantly lower upfront cost, reduced requirements for maintenance and less need for specialist (and expensive) IT support staff. As a result, small and medium sized businesses can focus more on what really distributes worth to their customers and results in financial returns. These, at least, have been the optimistic prophecies about Cloud Computing for the future. For example, Gartner ClO Agenda Report (2016) predicts that overall global spending on communal information technology and Cloud Computing could be approximately $\$ 250$ billion by 2016 .

\section{Cloud Computing - What are the Definitions?}

Alshamaila, Y. Y. [1] claimed defining Cloud Computing is not easy. Kim, W et al., [13], claimed that Cloud Computing definitions have been reformed several times in the past and will certainly reform in the near future. Raj, P. [22] mentioned that defining Cloud Computing is the key challenge for discussion due to the numerous background concepts and types of Cloud Computing service. Many scholars have defined Cloud Computing from the perspectives of various stakeholders such as scientists, providers, consumers, developers, engineers and service managers and from various applications aspects, but there is no clear consensus. The following table shows several different definitions of Cloud Computing based on both technical and service characteristics.

Table-1: List of Cloud Computing definitions (Compiled by the author)

\begin{tabular}{|c|c|}
\hline References & Definition of CC/ Quote \\
\hline $\begin{array}{l}\text { The National Institute } \\
\text { of Technology and } \\
\text { Standards (NIST) } \\
\text { [18] }\end{array}$ & $\begin{array}{l}\text { "Cloud Computing is a model for enabling convenient, on-demand network access to a shared pool of configurable } \\
\text { computing resources (e.g., network, servers, storage, applications, and services) that can be rapidly provisioned and } \\
\text { released with minimal management effort or service provider interaction". }\end{array}$ \\
\hline [21] & $\begin{array}{l}\text { "Cloud Computing is a style of computing where scalable and elastic IT capabilities are provided as a service to } \\
\text { multiple external customers using Internet technologies". }\end{array}$ \\
\hline [20] & $\begin{array}{l}\text { "Cloud Computing is a complex infrastructure of software, hardware, processing, and storage, all of which are available } \\
\text { as a service". }\end{array}$ \\
\hline [6] & $\begin{array}{l}\text { "Cloud Computing is a type of parallel and distributed system consisting of a collection of interconnected and } \\
\text { virtualised computers that are dynamically provisioned and present as one or more unified computing resource based } \\
\text { on service-level agreements established through negotiation between service provider and customers". }\end{array}$ \\
\hline [15] & $\begin{array}{l}\text { "Cloud Computing, in which not just our data but even our software resides within the cloud, and we access everything } \\
\text { not only through our PCs but also cloud-friendly devices, such as smart phones, PDAs...the mega computer enabled } \\
\text { by virtualisation and software as a service... this is utility computing powered by massive utility data centres". }\end{array}$ \\
\hline
\end{tabular}

\section{Cloud Computing - What are the Main Characteristics?}

Cloud Computing, which is a resource provisioning model, integrates a number of existing technologies that have been applied in grid computing, distributed systems, utility computing, service-oriented architectures, the 'internet of things', IT outsourcing and so on [17]. This section will explain the key characteristics of Cloud Computing and the differences between the features of technical, qualitative and economic business-related aspects of Cloud Computing.

\section{Cloud Computing - Key Characteristics}

- On-demand self-service through a secure portal: The cloud service user does on-demand self-service provisioning for server, network, and storage capabilities, without interacting with the service providers [11].

- Scalability and Elasticity: Rapidly scale the computing capabilities up or down, always elastically to maintain cost efficiencies [11].

- Pay per use: Capabilities are charged using a metered, fee-for-service or advertising-based billing model to promote optimization of resource use [11].

- Ubiquitous access: Capabilities are available over the network and accessed through standard mechanisms that promote use by heterogeneous thick, thin, or mobile client platforms. Thick client provides users with more features, graphics and choices making the platform more customizable. Thick client does not rely on a central processing server because normally processing is done locally on the user system and the server is accessed primarily for storage purposes [4]. But in contrast, a thin client communicates with a central processing server, where very little hardware and software is installed on the user's machine [4]. And, mobile or smart clients support work offline. Smart clients can work with data even when they are not connected to the Internet. These applications have the capability to be deployed and updated in real time over the network from a centralized server. Smart clients support multiple platforms and languages because they are built on web services and can run on almost any device that has Internet connectivity including desktops [4]. Services must be secure everywhere 
in the cloud, and access to the cloud through Internet devices must be secured to ensure data integrity, robust backups, authentication and cyber-attack protection [11].

- Location-independent resource pooling: Computing resources of the provider(s) are pooled to serve all users using a multitenancy model, with different physical and virtual resources dynamically assigned and reassigned according to user demand. No control or knowledge over the exact location of the provided resources is needed [11].

\section{Cloud Computing - Distinctions between technical, qualitative and economic aspects}

This section will describe and differentiate the technical, qualitative and economic characteristics of Cloud Computing.

\section{Technical Characteristics}

Technical characteristics are the base that supports other practical and financial requirements. Below I will summarise the technical aspects and their explanations.

\section{Virtualisation}

"Virtualisation is an important characteristic of Cloud Computing. It refers to a multi-layer hardware platform, operating system, storage device, network resources and so on" [17]. For example (so called) 'desktop virtualisation' provides a simulation of a user's experience of using applications, accessing online resources, communicating with other users as if they were operating their own desktop machine when, in reality, they may be interacting with multiple different platforms many of which are remotely located [7].

\section{Multi-Tenancy}

Multi-tenancy is a highly important issue for CC providers. It allows sharing of resources and costs across multiple users across a variety of platforms [17].

\section{Security}

Security is the biggest concern for adoption of Cloud Computing. In order to attract potential clients, CC providers must ensure sufficient security to protect clients' private data, communications and application use. CC providers must segregate data for different clients, and they need to ensure efficient replication and recovery mechanisms to provide robust recovery from failures [17].

\section{Programming Environment}

The programming environment is important for CC providers to be able to replicate many storage and application features across different computing platforms in order to exploit Cloud features. Much of this capability was developed during the earlier emergence of distributed computing in heterogeneous environments (see above, pp. 11). For example, the programming language 'Java ${ }^{\mathrm{TM}}$ ' (released by Sun Microsystems in 1995, Gosling et al., 1996) was designed explicitly to allow application developers to "write once, run anywhere" (WORA, Gosling, ibid.) on any platform capable of compiling Java code. The programming environment had to be capable of addressing issues such as cross-platform execution (differing operating systems), multiple administrative domains, resource heterogeneity (differing file formats), performance stability and robust exception handling in highly dynamic environments [17].

\section{Qualitative Characteristics}

This refers to qualities or properties of Cloud Computing, rather than specific technological characteristics. One qualitative characteristic can be realised in multiple ways depending on different providers. In the following, I am going to present the qualitative characteristics of Cloud Computing which in part define CC's scope and purpose.

\section{Elasticity}

Elasticity means the provision of services in elastic and adaptable ways, sufficient to allow users to request the service in near real-time without having to engineer new capabilities and resources at times of peak loads. The services required are measured in "fine-grain" detail, therefore, beforehand - with "Service Level Agreements" (SLAs) specifying the capacities and capabilities of the CC providers' services in advance. The terms of the CC provider's offering are then matched to a client's usage and set out in the SLA [17]. In terms of a CC provider's business model, thus, different levels of service can be offered at different prices - in relation to 'uptime' (uninterrupted service provision), storage requirements, access to applications, bandwidth (speed of communications) and numbers of users and devices to be supported [17].

\section{Availability}

This refers to a relevant capability that satisfies specific requirements of the outsourced services [17]. SLAs do not simply set out for client users the terms of a CC provider's offer. They have also to specify the conditions under which business clients may extend or add to their requirements over time - for example to accommodate new business partners or business expansion - together with the costs associated with such developments. From the business CC user's point of view, service availability conditions may require some internal re-engineering of business functions (together with negotiation of changed conditions, for example with suppliers), to adjust operations to the new services [17]. 


\section{Reliability}

Reliability signifies the ability to ensure constant system operation without disruption. Reliability is a particular Quality of Service (QoS) requirement, focusing on prevention of loss and robust recovery from errors and system failures [17].

\section{Agility}

Agility is a fundamental requirement for Cloud Computing. CC providers must be capable of on-line responses to changes in resource demand and environmental conditions. At the same time, efforts have to be made by clients to re-provision existing applications from an in-house infrastructure to Software as a Service (SaaS) vendors. Agility entails that both sides collaborate to provide self-management capabilities [17]. Agility is a possible problem for many small and medium sized businesses in Bangladesh. Lacking (as many do) sufficient IT skills and awareness, some fail to anticipate the internal readjustments needed to adopt CC solutions.

\section{Economic Characteristics}

Part of my argument, above, has been to support a view that the distinctive characteristics of Cloud Computing do not arise only from the technologies themselves which had been developed many years previously, but also from the characteristics of the business model which the technologies (in IT and in Network Communications) enabled.

Economic characteristics make Cloud Computing distinct, compared to other paradigms for ICT use in businesses. In a commercial environment, service offerings are not limited exclusively to the technological perspective but extend to a broader understanding of business needs. In the following, I am going to provide a summary of the different economic aspects of Cloud Computing.

\section{Pay-as-you-go}

Pay-as-you-go is a basic technique for the Cloud Computing business model, which means users need to pay according to the actual consumption of resources and services. Cloud Computing reduces the cost of infrastructure maintenance and acquisition, so it can help enterprises, especially small to medium sized. It may also reduce time to market and yield speedier return on investment [17]. For the CC Computing business user, cost effectiveness of CC adoption has to be factored into evaluation of potential benefits in terms of business expansion, increased capacity, speedier 'turnaround' times, potential to enter new markets.

\section{Operational Expenditure}

The infrastructure of CC operations is typically provided by a third party and does not need to be purchased for infrequent intensive computing tasks nor does not require extensive knowledge of IT. Therefore, it is easy for the users to enter the computing world [17]. In my own investigation, however, I will question whether (for SMEs in Bangladesh) initial operational expenditure is a critical factor in $\mathrm{CC}$ adoption. Adaptation of existing operational processes to accommodate $\mathrm{CC}$ has to be offset against the cost of adopting Cloud Computing solutions.

\section{Energy Efficiency}

Energy-efficiency is due to the ability of Cloud Computing services to reduce the consumption of under-used resources. Computers are administrated centrally, so additional costs of energy consumption as well as carbon emission can be better controlled than in other cases. Moreover, green IT issues are subject to both software stack and hardware level, where software stack is a group of programs that work in tandem to produce a result or achieve a common goal. It also refers to any set of applications that works in a specific and defined order toward a common goal, or any group of utilities or routine applications that work as a set [17].

\section{Cloud Computing - What are the Deployment Models?}

Cloud Computing has been developed based on the presence of public computer competences; additional deployment models dependent upon corporate location and volume of circulation have been espoused [19]. Cloud computing may be deployed in different fashions, depending on the intended scope of usage and the type of exclusive or non-exclusive approach to providing CC services to the service acceptors. I am classifying these different deployment models as follows based on a classification by [14].

- Public/External Cloud,

- Private/Internal Cloud,

- Hybrid Cloud and

- Communal Cloud

\section{Public/External Cloud Model}

According to [17] the public cloud is the typical cloud-computing example, in which a service provider makes resources, such as applications and storage, available to the general public over the Internet. Public cloud or external cloud describes Cloud Computing in the typical mainstream sense, and it is open to the public or to industry and owned and managed by a Cloud service provider [11]. Public Cloud has been defined by [2] as a "cloud made available in a pay-asyou-go manner to the general public". Examples of Public / External Cloud services would be Apple's iCloud service, Microsoft's Office 365 service and a variety of specialist services, like "DropBox" (file sharing), Instagram (photos, video sharing), and other 'social media' sites. The common characteristic of such services is that they are accessible via any web browser or smartphone App from anywhere - but can charge fees for storage of 
data and / or access to premium services. The business model is, thus, individual subscription-based together with revenue generated from advertising.

\section{Private/Internal Cloud Model}

The private / internal cloud defines an exclusive computing service that distributes access to a controlled number of people usually via interior networks or extranets that require authentication by remote users. Business organisations tend to prefer a private cloud service because of the need to retain accurate control over their data in order to obtain all the scalability, metering and nimbleness benefits of a public cloud without yielding control, security and usage costs to a service provider [17]. [8], identified that there are many reasons why an organisation may adopt a private cloud deployment model such as (a) there is a greater need to increase and enhance the exploitation of existing in-house resources, (b) service users are concerned about security, trust and privacy of their data, and (c) organisations look for total control over 'mission-control' activities, which are hosted behind their network firewalls and accessible only by authenticated devices and users.

\section{Hybrid Cloud Model}

According to [12], "A hybrid cloud uses a combination of public cloud, private cloud and even local infrastructures, which is typical for most IT enterprises". This deployment model enables data and application portability [11]. The objective of adopting a hybrid cloud is so that users can quickly switch from private cloud to public cloud in terms of shortage of capacity in the private cloud for extra resources to carry out their business operations [11]. In addition, a hybrid cloud may facilitate remote working ("working from home") for staff whose access needs to be limited (owing to security concerns). On the other hand, organisations may adopt a hybrid deployment model to optimise their resources in order to increase their core competencies by outsourcing peripheral business functions to the CC provider while controlling core activities in-house through their private network [8]. Hybrid models are a composite solution to distributing workload to staff depending upon cost and upon operational compliance factors. Users can deploy an application hosted on a hybrid infrastructure where some nodes are running on real physical hardware and others on cloud server instances [17]. Although a hybrid deployment model may be appropriate for businesses, which are already committed to legacy systems, which have to remain "in-house", the advantages of a fully CC-based solution are reduced [8].

\section{Community Cloud Model}

The community cloud model involves sharing by two or more organizations with shared concerns [10]. This model overlaps with 'computing grids' to some extent, in respect of different firms in a private community sharing a cloud based substructure. Within the community model, firms may have apprehensions about mission, security necessities, strategy, and acquiescence in all parties conforming to the 'community' requirements [17]. The community deployment model may be managed by an organisation or by a third party. This model may exist on premises or off premises [11]. This model can additionally be aggregated with a public cloud-based model (see above) to configure a 'cross-boundary' structure [17]. An example of a community model would be Terremark's Enterprise Cloud platform built by the United States Federal Government [17].

\section{Cloud Computing - What are the Service Models?}

Cloud Computing is characteristically a distribution of modified computing services over the Internet, which can be accessed in a simple and ubiquitous way [1]. Cloud Computing can co-operate with clients (users or applications) in multiple ways via serverclient or peer-to-peer network services [10]. According to [25], the service models can be classified in many ways through web based or customised interfaces such as:

- Software as a Service (SaaS)

- $\quad$ Platform as a Service (PaaS)

- Hardware as a Service (HaaS)

- Development/Database/Desktop as a Service (DaaS)

- Infrastructure as a Service (laaS)

- Business as a Service (BaaS)

- $\quad$ Framework as a Service (FaaS) and

- Organization as a Service (OaaS)

In essence, however, Cloud Computing services can be categorised into three key services based on the computing necessities of the clients and various levels of Cloud Computing substructure namely, Software as a Service (SaaS), Platform as a Service (PaaS) and Infrastructure as a Service (laaS) [25]. According to [18], these categories of Cloud service models are known from end-user's viewpoint - though a CC provider's viewpoint may incorporate various combinations of these services customised to different clients' needs.

In my evaluation of the benefits and barriers to CC adoption amongst Bangladeshi SMEs, my focus will be upon these three key service provisions (SaaS, PaaS, laaS) and the level of awareness SME owners and managers have about the potential benefits and limitations of their adoption (see Chapter 6), where other types of service, different from these three, may be appropriate, I will indicate the special circumstances within the SME context that may recommend these kinds of provision. 


\section{Software as a Service (SaaS)}

The Software as a Service model offers an on-demand cloud-based foundation for software, which is usually web-delivered content that clients access through a web browser. Software can be accessed in any of the Cloud deployment models [10]. Thus, clients do not necessarily need to install and run those applications on their own computers. These applications (e.g. document editing embedded in a web browser) can be used at any time from any location on the globe [18 - 8]. This service allows users to access reduced forms of desktop applications via the web. SaaS delivers applications as fully or partially isolated services, similar to desktop, standalone applications, with which users are already familiar. These applications can be in the form of web-based applications combined with non-remote applications - with Internet based storage or connectivity to other system communications' resources [25]. SaaS is a multi-tenant platform, which allows users to access software applications hosted by Cloud providers on a pay-as-you-go or subscription basis [25]. The advantages of SaaS services are straightforwardness of incorporation into business operations, familiarity, low-cost and scalability. In addition, the overheads associated with maintenance and upgrading are (in the main) borne by the CC provider, rather than the client. The disadvantages of SaaS are perceived to be the security issues (new users may not perceive it to be secure to store their firms' data on Cloud-based servers [10]. A second disadvantage may be a lack of compatibility of Cloud-based applications with legacy software, which may be costly to upgrade or replace.

\section{Platform as a Service (PaaS)}

Platform as a Service (PaaS) offers underlying hardware and operating system technologies, including virtual servers, file systems, database management systems, developer tools and network support for the CC users to install their own customised applications [10]. The platform providers within PaaS services reduce the need for clients to invest in and maintain their own servers, data-storage devices, software development environments and specialist network management utilities. In PaaS, instead of planning and costing regular upgrades to systems, developers focus on their own business-specific applications [10]. PaaS that is running via a CC provider's platform distributes resources such as Linux, Apache, MySQL and PHP to clients. This simplifies the distribution; maintenance and version management of applications with less cost and fewer complications than may be involved in purchasing and maintaining in-house hardware or software resources [11]. PaaS typically supports concurrent operators how to use applications by offering concurrency administration, scalability and safety therefore operators do not need to manage or control core Cloud substructures for deployment and hosting environment configurations [11]. Examples of PaaS inclues Cisco (WebEx connect), Amazon Web Services, Google, Windows Azure and Apple SDK toolkits available online [11].

\section{Infrastructure as a Service (laaS)}

Infrastructure as a Service (laaS) is an on-demand service of virtualisation for clients subscribing to CC services. The idea of Infrastructure as a Service is a transformation of Hardware as a Service (HaaS) to promote an all-inclusive approach to run IT substructures as services to end-users [10, 27]. The fundamental scheme of laaS is to create a flexible environment for clients to perform multiple tasks [6]. In laaS, designers have to set up and install their own operating systems, database management systems and all necessary specialist software to operate and manage hardware and software within their own operational environment [10]. laaS service models delivered to the clients including networking, computational, and storage resources to run different types of software specific to the CC client's needs. Using laaS, clients do not necessarily need to have control nor to build essential IT substructures, but they can have control of operating systems and their deployed applications [11]. Usages of the most current versions of technologies on a pay-peruse or subscription basis are the key advantage of laaS [11]. Some of the key providers of Cloud laaS providing fundamental IT hardware and software resources are Cisco, HP, IBM, Dell, VMware, Red Hat and Microsoft [11].

\section{Cloud Computing - What are the Key Drivers?}

The world is undergoing a digital and mobile revolution with large amounts of data, with rapid access via more media than ever before. Technology users have moved onto social media and mobile applications with the expectation that these will not only enhance their personal lives, but also enable them to work in different, more convenient ways and many trusts their technologies to be their main connotation arrangement [5]. Cloud Computing offers a new path for businesses to use the competences acquired from users' familiarity with digital technologies to meet clients' requirements and drive future development. About three-fourths of the firms globally had piloted, adopted or implemented Cloud Computing in their firms and $90 \%$ of them expected to adopt Cloud Computing services by 2015 [5]. In addition, firms who have already adopted Cloud Computing services are expected to have grown from $13 \%$ to $41 \%$ by 2015 [5]. Moreover, [5] also identified that a higher percentage of large firms (those with revenues above US\$20 billion) are testing Cloud-based services while small and medium sized firms are also experimenting with adoption. Furthermore, $67 \%$ of firms with less than US $\$ 1$ billion turnover and 76\% of the firms with revenues from US\$1 billion to US\$ 20 billion have been already adopted Cloud Computing services for some purposes, which indicates how the world Cloud Computing market is forecast to grow $22 \%$ yearly to US $\$ 241$ billion revenue by 2020 [5]. The data above suggests that the demand for Cloud Computing services is growing rapidly throughout the world's business communities. The concern of my investigation is to evaluate how "uneven" this demand may be depending upon regional, geographical and other economic factors, relative to the types and locations of the businesses concerned (especially SMEs). The major benefits or drivers for the adoption of Cloud Computing are listed and discussed below.

\section{Flexibility of Cost}

Flexibility of cost is a key reason for many firms to consider Cloud Computing adoption in the first place [5]. Cloud Computing offers an ability to reduce fixed IT costs and to move to a 'pay-as-you use' cost structure [5]. By enabling movement of 
capital expenditure to operational expenditure or from fixed to variable expenditure Cloud Computing may support a company in reducing fixed IT cost. Moreover, with computing applications, there is no longer a need to invest in specialist hardware or software or to pay frequent software update fees. Firms only need to pay what they use and when they may need to increase usage. This facility offers flexibility and eradicates the need for initial capital expenditure [5].

\section{Scalability of Business}

Scalability of business operations or IT scalability is recognized by many firms as one of the major drivers for adoption of Cloud Computing. Moreover, Cloud Computing services, it is claimed [5], allow companies both to scale up and, in less favourable economic circumstances, to scale down their costs in relation to IT provision - unlike inhouse IT provision which requires on going expenditures even when under-used.

\section{Market Adaptation}

Market adaptation in today's economic environment is vital because ability to respond to rapidly changing purchaser needs is one of the key differentiators. Therefore, firms are continuously looking for a path to develop their speed of response to market demands [5]. By empowering businesses to adapt procedures, services and products to meet the changing needs of the market, Cloud Computing has the ability to enable prototyping and innovation, so may support more rapid times to market [5]. Of course, rapid adaptation to changing market conditions applies primarily to market sectors, which are more turbulent. [5] that Cloud Computing services provide easier ways in which companies can react to rapid market changes has been little tested. Cloud Computing providers have also to accommodate fairly rapid change in technologies, pricing structures, service provision and in their own hardware and software infrastructures in order to adapt to changing market conditions. Such changes may threaten the adoption of Cloud-based services if providers lack the capacity to react to changes in technologies, usage, connectivity and costs.

\section{Masked Complexity}

Cloud computing not only offers business scalability and market adaptability but also offers the benefits of masking complexity. Cloud Computing has a way to hide parts of the technical intricacy of its operations from clients. This re-distributes the skills needed for end-users to be productive in their use of business-specific IT. It also offers the promise of reducing training costs for new employees. Rather, IT specific skills (configuring user accounts, maintaining robust and reliable data storage, ensuring optimal network 'uptime', and others) are transferred (on the whole) to CC providers [5]. Complexity is 'masked' from the clients (and from their customers) enabling, a business to increase the sophistication of its services and products without incurring as much overhead in respect of end-users' knowledge or technical facility to manage, maintain and control the technical infrastructure needed. For example, software updates and maintenance can be completed in the 'background' without the involvement of the consumers' [5]. However, it should be noted that - especially in CC adopting businesses whose core activities require extensive interaction (B2B or B2C) with customers - 'masking complexity', in this sense, requires a separation of the businesses' product or service-specific support (for example, product specifications or pricing / payment options) from IT service support (since this will usually be located elsewhere - in the CC service provider's support activities. When customers' requirements involve a combination of product / service specific support with IT service support, this separation may degrade the quality of support provided. A concern for quality of service, which for many SMEs may be an important differentiator in the market.

\section{Context-driven Variability}

Context-driven variability is one of the drivers of Cloud Computing due to expanded computing power and capacity varied for each business user's requirements. Users want to keep their business-specific data based on their own operational requirements. Cloud Computing services offer storage facilities for clients for their operational requirements, which permits product or service customization [5]. Context-driven variability is a significant Cloud attribute. In principle, CC services ought to be able to tailor services to the specific requirements of business subscribers. In reality, this (for SMEs in particular) may be an illusion. Larger scale CC service providers (Microsoft, Apple, Google) in general do not offer customised services. The onus is upon the business user to tailor their business processes to make most effective use of generic offerings like iCloud, MS Office 365, Google Plus. In theory, customisable CC services should allow for more user-centric involvement and address-fragmented consumers' preferences for the companies subscribing [5]. In reality, "context-driven variability" is rare for businesses signing up for generic CC services.

\section{Connectivity}

Connectivity is claimed to be one of the business enablers and key benefits of Cloud Computing offerings [5]. Cloud computing simplifies the exterior relationship with associates and customers, which can lead to enhancements in efficiency and amplified novelty. Berman, S et al., [5], point, here, appears to single out the potential for Cloud-based services to unify modes of interaction with both 'upstream' (supplier networks) and 'downstream' (customer relationship management) functions within a business. The underlying assumption appears to be that if sufficient numbers of businesses and customers subscribe to the same CC service, then connectivity and interaction will be simplified and enhanced. The assumption is questionable: integrating suppliers, businesses and customers into a 'single' CC ecosystem (as I will argue) requires considerably more than these services (at present) can provide. What, for example, is the incentive for suppliers (or customers) to change their modes of interaction with a business to accommodate the services that a CC provider may enable? 


\section{Facilities Consolidation}

Cloud computing services offer consolidation facilities. These may appear to be attractive to many companies for the saving implied for their data resources. It is claimed that resources such as storage, computational access, and memory and network bandwidth can be pooled under Cloud facilities in a way that is cheaper than localised provision. Consolidation of data storage, ease of access from anywhere to shared data, reliability of recovery from data loss: all of these may constitute drivers in favour of CC service adoption.

\section{Labour Optimization}

According to [16], "Cloud Computing deployment does not require as much provisioning, software development, or maintenance as a conventional infrastructure, organizations can make better use of valuable ICT expertise by redirecting the workforce from routine operational and maintenance duties to mission-critical tasks". Every firm expect to be able to save labour cost. By adopting Cloud Computing services and using its facilities businesses may save large amounts of labour cost by using their IT specialists to meet specific requirements instead of using them for generic IT related tasks within the business [16].

\section{Swift Deployment}

In Cloud environment, there is no longer need a software installation or configure the system, which gives huge benefits of the consumers as most of the consumers are not efficient of setting up hardware and installing software for their system. Cloud Computing offers a very swift deployment facilities for the consumers [16].

\section{Increased Flexibility}

Cloud Computing has various deployment models and services, which gives a firm to choose their specific model and service or combination of many. This service is one of the key drivers for Cloud Computing because it guarantees the implementations can be associated thoroughly with industry requires and ICT policies [16].

\section{Assured Service Level}

Cloud computing can assure and offer a better level of services than other ICT groups provides limited resources. With the proper combination of Cloud models, a firm can ensure the existing service-level agreements (SLA) are sustained [16].

\section{Robust Resilience}

Cloud Computing offer automated recovery or disaster recovery facilities, which is easy to access by the consumers due to its consolidated resources. This is another top Cloud driver as some point firms use their internal Cloud model as a failover for public Cloud to increase the flexibility. Due to the dynamic flexibility of Cloud Computing, firms are able to responds to service outage very quickly comparing internal ICT specialist [16].

\section{Cloud Computing - What are the Key Barriers/Challenges}

Although there are significant drivers for adoption of Cloud Computing, there are still some noteworthy challenges. Ghaffari, $\mathrm{K}$ et al., [9] found in their research that security is a major concern of Cloud Computing adoption because of fears about online data security. Security is not the only barrier to adoption. There are some significant hurdles such as temporary loss of service (outage), ability to change suppliers (interoperability) and reliability, which are also key barriers to Cloud Computing adoption [26]. The following will describe in more detail the inhibitors to adoption of Cloud Computing.

\section{Reliability}

At present, enterprise-wide applications in larger businesses are so extensive that they have to be reliable and available to all requiring their provision [9]. In terms of systems failure, a recovery plan must begin with minimal disruption and additional cost. However, larger businesses may be able to invest in recovery systems mitigating the effect of systems failure, but small businesses typically do not have such resources. So, it is essential to monitor a track record of reliability to inform adopters of the need to monitor systems reliability in order to encourage the wide-ranging adoption of Cloud Computing [9].

\section{Security and Privacy}

A Cloud Computing approach presents a novel delivery model for IT solutions, which seems not to offer sufficiently high a level of security (of data) for enterprises [9]. Zissis, D et al., [26] claimed that there is a lack of sufficient certainty and security accompanied by the advent of every new technology or innovation. They [26] suggest it is unavoidable. The capability of Cloud Computing to address sufficiently privacy regulations has been called into question. Thus, firms have to address a multitudinous set of pre-requisites to safeguarding the privacy of individual's information [9].

\section{Open Access and Connectivity}

The realisation of Cloud Computing is dependent upon the availability of high-speed access to all and open access to computing resources similar to water and electricity power accessibility. However, in real life, connectivity and open access of Cloud Computing is not present globally [9]. Lack of open access and connectivity ought, therefore, to be a consideration for SMEs within their own business contexts: is there sufficient connectivity and access for their current and proposed supplier- and customer base? 


\section{Interoperability}

In the process of Cloud Computing adoption, it is pivotal to secure an appropriate level of interoperability between private and public clouds [9]. A large number of companies have made remarkable progress toward standardising their processes, data, and systems by means of implementation of ERPs. Standardisation requires an extensible infrastructure, which results in a fully integrated interoperability amongst instances [9]. SaaS applications delivered via the cloud provide a low capital, fast deployment option. Multiplatform interoperability may be important considerations for larger enterprises supporting a variety of IT platforms and a variety of means of access to online resources. It is not clear, however, that such considerations are relevant to SMEs. Depending upon the application, it is important to integrate with traditional applications resident on a separate cloud or upon local technologies. Standards can be an enabler or an impediment to interoperability in that existing ('legacy') systems may or may not inhibit the transfer to Cloudbased applications. The key resides in data integration and cross-platform compatibility [26]

\section{Economic Value}

It sounds obvious that by sharing resources to smooth out peaks, paying only for what is used, and cutting up-front capital investment in employing IT solutions the economic value of CC adoption will be there [9]. There is a necessity for SMEs to balance accurately all costs and benefits arising from Cloud Computing adoption -in both the short and medium term.

Hidden costs could encompass support, disaster recovery, application modification, and data loss insurance. Since usage expands and interoperability requirements for business processes become more complex, a novel approach is required [9]. Although accurate costings are difficult to compile, in my research I will focus upon the 'fears about cost', which SMEs in Bangladesh may have.

\section{Political Issues and Global Boundaries}

In the Cloud Computing world, there is variation in terms of where the physical data resides, where processing takes places as well as from where the data is accessed [9]. Given this variability, privacy rules and regulation may become problematic. Owing to the variable nature of rules and regulations in different political contexts, the direct involvement of government become more salient in standardizing and regulating the adoption process for Cloud Computing - both for providers and for purchasers [9]. An example, here, would be useful: e.g. (hypothetically) if an American owned private healthcare company operating in the UK transfers patient data to New York, predominantly for the (legal) use of that data in evaluating treatment costs / epidemiological trends, then it may become an issue whether Data Protection principles from UK Law (the Data Protection Act (as amended, 1998)) should apply to that data or whether State Law in New York (notably 'weaker' than UK legislation about how data may be used or communicated to third parties) should apply.

\section{CONCLUSION}

This paper aimed to identify various aspects associated with Cloud Computing including different advantages and disadvantages. Inevitably, Cloud Computing can offer numerous advantages to different stakeholders. However, Cloud Computing does have some challenges as well. It is notable that with the support of deployment and service models of Cloud Computing, challenges can be either minimised or eliminate because dynamic characteristics of Cloud Computing can always supports users.

\section{REFERENCE}

1. Alshamaila, Y. Y. (2013). An empirical investigation of factors affecting Cloud Computing adoption among SMEs in the North East of England. Newcastle University Business School, Newcastle upon Tyne. [Retrieved from: $<$ https://theses.ncl.ac.uk/dspace/bitstream/10443/2080/1/Alshamaila\%2013.pdf> Accessed on 23/12/2015]

2. Apulu, I., \& Latham, A. (2009). Information and communication technology adoption: Challenges for Nigerian SMEs, TMC Academic Journal, 4(2), 64-80.

3. Aravind, R. G., Sampath, M. K., \& Venkatesh, V. G. (2013). Opportunities for Cloud based Software as a Service (SaaS) Warehouse Management System: An Indian Industry Insight. SIBM, 4(2), 43-54.

4. Banerjee, P. (2017). An intelligent IT infrastructure for the future", Proceedings of 15th International Symposium on Highperformance Computer Architecture, HPCA, Raleigh, NC, USA.

5. Berman, S., Marshall, A., \& Srivathsa, R. (2012). The power of cloud-Driving business model innovation, IBM Institute for Business Value.

6. Buyya, R., Broberg, J., \& Goscinski, M. A. (2011). Cloud Computing: Principles and Paradigms. Hoboken, New Jersey: John Wiley and Sons.

7. Cafaro, M., \& Aloisio, G. (2010). Grids, clouds and virtualization. Springer Publishing Company, Incorporated, 1st edition

8. Dillon, T., Chen, W., \& Chang, E. (2010). Advanced Information Networking and Applications (AINA), 2010 24th IEEE International Conference on. 20-23 April 2010

9. Ghaffari, K., Delgosha, S. M., \& Abdolvand, N. (2014). Towards Cloud Computing: A SWOT Analysis on its adoption in SMEs. International Journal of Information Technology Convergence and Services (IJITCS), 4(2).

10. Jamsa, K. (2013). Cloud Computing SaaS, PaaS, laaS, Virturalization, Business Models, Mobile, Security, and More. Jones \& Bartlett Learning, United States of America, 5-15.

11. Josyula, V., Orr, M., \& Page, G. (2012). Cloud Computing Automating the Virtualized Data Center. Cisco Press, Unite States of 
America, 9-16.

12. Khan, I. (2015). Why Business (SMEs) Should Adopt Cloud Computing. Business Information Technology, Oulu University of Applied Sciences [Retrieved from: <https://www.theseus.fi/bitstream/handle/10024/101464/Khan_Imran.pdf?sequence=1> Accessed on 04/02/2016]

13. Kim, W., Soo Dong, K., Eunseok, L., \& Sungyoung, L. (2009). Adoption issues for cloud computing, Proceedings of the 7th International Conference on Advances in Mobile Computing and Multimedia. Kuala Lumpur, Malaysia. ACM.

14. Kundra, V. (2010). State of Public Sector Cloud Computing, US Chief Information Officers Council [Retrieved from: $<$ www.cio.gov/documents/StateOfCloudComputingReport-FINALv3_508.pdf> Accessed on 19/07/2016]

15. Low, C., Chen, Y., \& Wu, M. (2011). Understanding the determinants of cloud computing adoption. Industrial Management \& Data Systems, 111(7).

16. Macias, F., \& Thomas, G. (2011). Cloud Computing Advantages in the Public Sector. How Today's Government, Education, and Healthcare Organizations Are Benefiting from Cloud Computing Environments [Retrieved from: < https://www.cisco.com/c/dam/en_us/solutions/industries/docs /c11-687784_cloud_omputing_wp.pdf> Accessed on: 10/09/2019]

17. Magoulès, F., Pan, J., \& Teng, F. (2013). Cloud Computing Data-Intensive Computing and Scheduling. CRC Press, Taylor \& Francis Group, 6000 Broken Sound Parkway NW, Suite 300, Boca Raton, FL 33487-2742, 1-17.

18. Marston, S., Li, Z., Bandyopadhyay, S., Zhang, J., \& Ghalsasi, A. (2011) 'Cloud computing - The business perspective', Decision Support Systems, 51(1), 176-189.

19. Mell, P., \& Grance, T. (2010). The NIST Definition of Cloud Computing [Retrieved from < http://www.newinnovationsguide.com/NIST_Cloud_Definition.pdf> Accessed on 09/11/2015]

20. Misra, S. C., \& Mondal, A. (2010). Identification of a company's suitability for the adoption of cloud computing and modelling its corresponding return on investment. Mathematical and Computer Modelling, 53, 504-521.

21. Plummer, D., Cearley, D., \& Smith, D. (2008). Cloud computing confusion leads to opportunity. Technical Report G00159034, Gartner Research.

22. Raj, P. (2013). Cloud Enterprise Architecture. CRC Press Taylor \& Francis Group, Great Britain. 231-236

23. Staten, J. (2009). Hollow Out The MOOSE: Reducing Cost With Strategic Rightsourcing.

24. Velte, T., Velte, A., \& Elsenpeter, R. (2009). Cloud computing, a practical approach. McGraw-Hill, Inc

25. Weinhardt, C., Anandasivam, D. I. W. A., Blau, B., Borissov, D. I. N., Meinl, D. M. T., Michalk, D. I. W. W., \& Stober, J. (2009). Cloud computing-a classification, business models, and research directions. Business \& Information Systems Engineering, 1(5), 391-399.

26. Zissis, D., \& Lekkas, D. (2012). Addressing Cloud Computing Security Issues: Future generation Computer Systems, 28(3), 583592.

27. Sotomayor, B., Montero, R. S., Llorente, I. M., \& Foster, I. (2009). Virtual infrastructure management in private and hybrid clouds. IEEE Internet computing, 13(5), 14-22. 